\title{
To Be a Realist about Quantum Theory
}

\author{
Hans Halvorson
}

January 19, 2018

\section{Introduction}

There's a story that some philosophers have been going around telling. It goes something like this:

The pioneers of quantum mechanics - Bohr, Heisenberg, Dirac, et al. - simply abandoned hope of providing a realist theory of the microworld. Instead, these physicists settled for a calculational recipe, or statistical algorithm, for predicting the results of measurements. In short, Bohr et al. held an antirealist or operationalist or instrumentalist view of quantum theory. ${ }^{1}$

Implicit in this story is a contrast with the "traditional aspirations of science" to describe an observer-independent reality. Having built up a sense of looming crisis for science, the story-teller then introduces us to the heroes, those who would stay true to the traditional aspirations of science.

As the 20th century moved into its second half, there arose a generation of renegade physicists with the courage to stand up against antirealism and operationalism. These valiant men David Bohm, Hugh Everett, John Bell - renewed the call for a realist theory of the microworld.

This kind of story can be very appealing. It's the age old "good guys versus bad guys" or "us versus them" motif. And those "ist" words make it easy

\footnotetext{
${ }^{1}$ For example: "the uncertainty principle is connected with certain antirealist ideas of Heisenberg and Bohr and the statistical interpretation of the wave function" (Ney and Albert, 2013, pp 14-15).
} 
to distinguish the good guys from the bad, sort of like the white and black hats of the classic westerns.

The story is brought into clearer focus by talking about the quantum wavefunction. What divides the realists from the antirealists, it is said, is their respective attitudes toward the wavefunction: antirealists treat it as "just a bookkeeping device", whereas realists believe it has "ontological status". Witness the faux-historical account of Roger Penrose:

It was part of the Copenhagen interpretation of quantum mechanics to take this latter viewpoint, and according to various other schools of thought also, $\psi$ is to be regarded as a calculational convenience with no ontological status other than to be part of the state of mind of the experimenter or theoretician, so that the actual results of observation can be probabilistically assessed. (Penrose, 2016, p 198).

I suppose that Penrose can be forgiven for oversimplifying matters, as well as for propagating the myth of the "Copenhagen interpretation" (see Howard, 2004). After all, there can be great value in simple fictional tales if they get readers interested in the issues.

I also imagine that Sean Carroll is aiming to generate some heat - rather more heat than light — when he poses the following dilemma about the wavefunction:

The simplest possibility is that the quantum wave function isn't a bookkeeping device at all ...; the wave function simply represents reality directly. (Carroll, 2017, p 167)

This seemingly simple dilemma — ontological status: yes or no? — is a fine device for popular science writing, which shouldn't demand too much from the reader. But is it really the right place to locate a pivot point? Is the question "ought I to commit ontologically to the wavefunction?" the right one to be asking?

Popular science writers aren't the only ones to have located a fulcrum at this point. In fact, some philosophers say that if you're a scientific realist, then you're logically compelled to accept the Everett interpretation. I'm thinking of this kind of argument:

If you're a realist about quantum theory, then you must grant ontological status to the quantum state. If you grant ontological 
status to the quantum state, and if quantum mechanics is true, then unitary dynamics is universal. Under these conditions, realism and unitary dynamics, you have two options: either you accept the completeness of quantum theory, or you don't. And if you accept the completeness of quantum theory, then the Everett interpretation is true.

In short, we are told that the following implication holds:

Realism + Pure QM $\Longrightarrow$ Everett

Notice how much work realism is supposed to do in this implication!

You might accuse me of caricature, and I'm sure I have left out much of the nuance in this argument. And yet, Everettians regularly gesture in this direction. For example, Wallace (2013) claims that the Everett interpretation "is really just quantum mechanics itself understood in a conventionally realist fashion," and that "there is one pure interpretation which purports to be realist in a completely conventional sense: the Everett interpretation" (Wallace, 2008). Similarly, Saunders claims that if we don't think of the wavefunction as a measure of our ignorance, then

the only other serious alternative (to realists) is quantum state realism, the view that the quantum state is physically real, changing in time according to the unitary equations and, somehow, also in accordance with the measurement postulates. (Saunders, 2010)

In short, if you're a good realist, then you'll say that the quantum state is physically real, and from there it's a short step to the Everett interpretation.

There is something strange about this sort of argument. The notion of "realism" is doing so much of the work - and yet, nobody has told us what it means. How could the "if realism then Everett" argument be valid when "realism" hasn't been defined clearly? And how could the argument be convincing when realism has not been motivated, except through its undeniable emotional appeal?

In this paper, I'll take a closer look at the distinction between between realist and antirealist views of the quantum state. I argue that this binary classification should be reconceived as a continuum of different views about which properties of the quantum state are representationally significant. What's more, the extreme cases - all or none - are simply absurd, and should be rejected by all parties. In other words, no sane person should 
advocate extreme realism or antirealism about the quantum state. And if we focus on the reasonable views, it's no longer clear who counts as a realist, and who counts as an antirealist. Among those taking a more reasonable intermediate view, we find figures such as Bohr and Carnap - in stark opposition to the stories we've been told.

\section{Extremists}

Suppose that you were asked to list historical figures on two sheets of paper: on the first sheet, you're supposed to list REALISTS (about the quantum state), and on the second sheet you're supposed to list ANTIREALISTS (about the quantum state). Suppose that you are asked to sort through all of the big names of quantum theory - Bohr, Heisenberg, Dirac, Bohm, Everett, etc..

I imagine that this task would be difficult, and the outcome might be controversial. For almost none of these people never explicitly said, "I'm a realist" or "I'm an antirealist" or "the wavefunction has ontological status" or anything like that. You'd have to do quite a bit of interpretative work before you could justify assigning a person to one of the lists. You'd have to assess that person's attitude toward the quantum state by studying their behavior and utterances with respect to it. For example, if person $X$ makes free use of the collapse postulate, with no proposed physical mechanism, then you might surmise that $X$ is either a mind-body dualist, or an operationalist about the quantum state, or both. In other words, an operationalist stance might serve as the best explanation for $X^{\prime}$ 's utterances and behavior.

The task of sorting people into REALIST and ANTIREALIST would be simpler for contemporary figures, who seem happy to embrace one of these two labels. For example, Sean Carroll and Lev Vaidman will tell you, with great passion, that the wavefunction is just as real as - in fact, more real than! - a rock, or a tree, or your spouse. In contrast, Carlo Rovelli speaks of the wavefunction as Laplace spoke of God: je n'avais pas besoin de cette hypothèse-là. And these are just a few examples among the many philosophers and physicists who have openly labelled themselves as realist or antirealist about the quantum state. Self-identified state realists include Esfeld, Goldstein, Ney, Saunders, Wallace, Zanghi, etc.. Self-identified state antirealists include Bub, Fuchs, Healey, Peres, etc.. The battle lines have been clearly drawn, but what is at stake? 
The right wing extremists say: quantum wavefunctions are things. That view is silly. The left wing extremists say: quantum wavefunctions are just bookkeeping devices. That view is just as silly.

\subsection{Right wing extremists}

One might think that the litmus test for realism about quantum theory could be posed as:

Do you believe that the wavefunction (more generally, the quantum state) exists?

Or as Callender (2015) puts it,

Is the quantum state part of the furniture of the world?

So when (Carroll, 2017, p 142) says that, "the basic stuff of reality is a quantum wave function", he's declaring his allegiance to wavefunction realism.

But what is this wavefunction thingy? Should I be thinking about it like I think about chairs or tables? No, say the philosophers; you have to be a bit more sophisticated about it. The preferred ontological reading of the wavefunction is as a field, on analogy to things like the magnetic field that surrounds the earth. Thus, to push the ontological picture further, things are represented by points in the domain of that field, and the properties of those things are the values of that field. What then are the things according to this ontological view? Some philosophers say that the wavefunction is a field on configuration space (Albert, 1996; Ney, 2012; North, 2013), so that the things are points of configuration space. Others say that the wavefunction is a multi-field on physical space (Forrest, 1988; Belot, 2012; Chen, 2017), so that the things are spacetime points.

These straightforwardly ontological views have been subjected to many criticisms (see Belot, 2012; Wallace and Timpson, 2010). Here I want to raise another kind of objection. Or rather, I want to make a request of the $\psi$-field theorists: would you please describe your theory clearly, including its states, properties, and the relationship between them. To my mind, the attraction of $\psi$-field theories is due in large extent to the vague, realist associations that they conjure up in our heads. "The wavefunction is a thing with a definite shape!" I wager that such theories are plausible only to the extent that it is unclear what they are really saying. 
For starters, in quantum theory, the primary theoretical role of the wavefunction $\psi$ is as a state. The $\psi$-field theorists ask us to change our point of view. Instead of thinking of $\psi$ as a state, we are to think of $\psi$ as a field configuration. There are numerous problems with this proposal.

In classical physical theories, the word "state" is shorthand for "a maximally consistent list of properties that could be possessed by the system simultaneously", or equivalently, "an assignment of properties to objects". In that case, there are two possible things we could mean by the sentence "the state $\sigma$ exists". First, we could mean that the list of properties exists. But this list is an abstract mathematical object, which would exist whether or not the corresponding theory is true. So, in this first sense, " $\sigma$ exists" is not interesting from the point of view of physics. Second, we could use " $\sigma$ exists" as an obscure shorthand for " $\sigma$ is the actual state", which in turn is shorthand for saying that certain other objects have certain properties. Thus, in this second case, " $\sigma$ exists" is cashed out in terms that don't refer to $\sigma$ at all. In philosophers' lingo, " $\sigma$ exists" is grounded in facts about other objects, and so isn't really about $\sigma$ at all.

Now, the defender of quantum state realism might simply say: "that was classical physics. In quantum physics, the state takes on a new role." I certainly accept that quantum physics changes some of the ways we talk about the physical world. But I'm not so sure that it makes sense to reify states. According to the normal senses of "object" and "state", we affirm that objects can be in states. Thus, if states are objects, then states themselves can be in states. But then, to be consistent, we should reify the states of those states, and these new states will have their own states, ad infinitum. In short, if you run roughshod over the grammatical rules governing the word "state", then you can expect some strange results.

To continue that line of thought, we assume that things can be counted. In other words, it makes sense to ask, "how many things are there?" But then, if states were things, it would make sense to ask, "how many states are there?" But now I'm completely puzzled. According to quantum theory, the universe has an infinite number of potential states, but only one actual state. What in the world would explain the absence of all the intermediate possibilities? Why couldn't there have been seventeen states? And what's more, why do physicists never raise as an empirical question, "how many states are there?" The reason is simple: physicists don't treat states as they do things, not even in the extended sense where fields also count as things.

I hope that by this stage you are at least partially convinced that it does 
violence to the logic of physical theories to talk about states as if they were things. But then you should agree that the role of a wavefunction is not to denote an object. Moreover, if $\psi$ does not denote a physical object, then the properties of $\psi$ do not directly represent the properties of a physical object. Granted, we should be careful with this latter claim. Even in classical physics, the properties of a state can represent, albeit indirectly, the properties of a physical object. For example, for a classical particle, "being in subset $\Delta$ of statespace" is a property of states that represents a corresponding property of the relevant particle. Nonetheless, there are two different types of things here - the particle, which is a concrete physical object, and its state, which is an abstract mathematical object. The latter tells us about the former, but should not be conflated with it.

In classical theories, there is also a sharp distinction between instantaneous configurations and states. If a configuration is represented by a point in the manifold $M$, then a state is represented by a point in the cotangent bundle $T^{*} M$. In many scenarios, $T^{*} M$ looks like a Cartesian product $M \times M$, where the first coordinate gives instantaneous configuration, and the second component gives momentum. In every case, there is a projection mapping $\pi: T^{*} M \rightarrow M$, and the preimage of any particular configuration $q \in M$ is an infinite subset of $T^{*} M$. But now, if $\psi$ is both a state and a field configuration, then it's unclear where it lives. Does $\psi$ live in the space $M$ of configurations, or does it live in the space $T^{*} M$ of states? How can it do both jobs at the same time?

These considerations show that the $\psi$-field view stretches the logic of classical physics beyond the breaking point. To treat $\psi$ as representing a field configuration is to disregard its primary theoretical role as a state. Or, at the very least, to treat it thus would obscure the difference, central to classical theories, between configurations and states.

If that isn't enough trouble for $\psi$-field views, we can also ask them to give an account of the properties that are possessed by this thing, the $\psi$-field. Recall that in a classical theory with statespace $S$, properties are typically represented by subsets of statespace $S .^{2}$ Then we say that the system has property $E \subseteq S$ just in case it is in state $\sigma \in E$.

Now, $\psi$-field theorists would like us to think of quantum theory on the model of a classical field theory. In this case, the statespace would be the

\footnotetext{
${ }^{2}$ We might require that these subsets be measurable or something like that. But that point won't matter in this discussion.
} 
space $C^{\infty}(X)$ of smooth complex-valued solutions to some field equation, and subsets of $C^{\infty}(X)$ would represent properties that the system can possess. ${ }^{3}$ For example, for any field state $f \in C^{\infty}(X)$, the singleton set $\{f\}$ represents the property of being in state $f$, and its complement $C^{\infty}(X) \backslash\{f\}$ represents the property of not being in state $f$.

For the purpose of performing certain calculations, a classical field theorist might complete $C^{\infty}(X)$ relative to some norm, obtaining a Hilbert space such as the space $L_{2}(X)$ of (equivalence classes of) square-integrable functions on $X$. The elements in $L_{2}(X)$ are no longer smooth functions, and in fact, they aren't really functions at all - they are equivalence classes of functions under the relation: $f \sim g$ just in case $\int|f-g| d \mu=0$.

In contrast, for a quantum theorist, $L_{2}(X)$ is simply one instance of, or one representation of, a Hilbert space $H$ of countably infinite dimension. Any two Hilbert spaces of the same dimension are isomorphic, and so it doesn't matter (for the physics) which one we choose. The states of the system are represented not by points in $L_{2}(X)$, but by rays. And the properties of the system are represented not by subsets of $L_{2}(X)$ but by closed subspaces. Thus, in short, while $L_{2}(X)$ is used by both the classical field theorist and the quantum mechanic, it is used in completely different ways in the two cases. For those of us who believe that the Hilbert space formalism is intended to represent reality, we could say that it represents reality in a very different way than a classical field theory does.

The $\psi$-field views ask us to forget the differences between quantum mechanics and classical field theories. But it won't be easy to forget these differences without doing violence to the representational role of the various pieces of the Hilbert space formalism. A classical theory comes with representatives (subsets of statespace) for many properties that are not represented in the Hilbert space formalism. The $\psi$-field theorist wants to lay claim to all these properties - for this seems to provide the coveted "god's eye view" of reality. In effect, the $\psi$-field picture is designed to make us feel like we've evaded the Kochen-Specker theorem. For, if physical properties are represented by subsets of $L_{2}(X)$, or by the mathematical properties of a function $\psi \in L_{2}(X)$, then each such property is either definitely possessed or definitely not possessed when the system is in state $\psi$. This view is intended to hide (or ignore? or deny?) the fact that a quantum state does not answer

\footnotetext{
${ }^{3}$ I'm ignoring here the fact that classical field theories would typically use the space of real-valued functions.
} 
all questions about which properties are possessed.

What's more, the $\psi$-field view only follows classical physics as far as giving an instantaneous snapshot of the possessed properties. As soon as it comes to drawing inferences about the system, it imposes ad hoc rules to block fallacious inferences. For example, in a classical field theory, if $\sigma$ and $\sigma^{\prime}$ are distinct field states, then knowing that the system is in state $\sigma$ permits you to assert that the system is not in state $\sigma^{\prime}$. Or in probabilistic terms, the probability of $\sigma^{\prime}$ conditional on $\sigma$ is 0 . If you carry that inference rule over directly into quantum theory, then you'll make false predictions. A Gaussian function $\psi$ centered at 0 is a different field state than a Gaussian function $\psi^{\prime}$ centered at 0.01 . Thus, on a classical picture, the property $E$ of "being in state $\psi$ " is inconsistent with the property $E^{\prime}$ of "being in state $\psi^{\prime}$ ", and $\operatorname{Pr}\left(E^{\prime} \mid E\right)=0$. But quantum theory says that $\operatorname{Pr}\left(E^{\prime} \mid E\right) \approx 1$.

Of course, $\psi$-field theorists are too clever to fall into the trap of carrying classical inference rules into the quantum domain. Although they purport to view the $\psi$-field classically, they stop short when it comes to reasoning about it. For the purposes of reasoning and making predictions, they turn to the Hilbert space formalism to guide them. Thus, we might summarize the attitude of these $\psi$-field views in a phrase: you can look at the world from the god's eye point of view; just don't reason about it as god would.

\section{$2.2 \quad$ Left wing extremists}

At one extreme, we have people telling us that the wavefunction is part of the furniture of reality. At the opposite extreme, we have people telling us that the wavefunction is "a mere calculational device" (Rovelli, 2016, p 1229), and that "it is mistaken to view the universal wave-function as a beable" (Healey, 2015). This second group of extremists is a curious bunch. They protest loudly against the wavefunction, producing elaborate (and interesting) arguments against its ontological status. And yet, they can't seem to live without it. In their books and articles, they accord a privileged role to the wavefunction. When they want to say something true about a quantum system, they consult the wavefunction before anything else. It makes one wonder: if they don't believe in the wavefunction, then why do they grant it a special role in their representations of reality?

The practice in physics, followed by realists and antirealists alike, is that each classically described "preparation" or "experimental setup" may be represented by a unique quantum state. In fact, the ability to associate quantum 
states to classically described experiments is one of the skills that displays mastery of quantum theory. Once an experiment has been adequately described, then there is no remaining latitude for idiosyncratic or subjective state assignment. There is just one correct state, as will be borne out by checking the statistics of measurement outcomes. The fact that physicists have correctness standards for quantum state assignments strongly suggests that they grant these states some sort of representational role.

Healey (2017) makes exactly this point, and he uses it to make an argument for quantum state objectivism: i.e. the belief that there are objectively correct ascriptions of quantum states to physical systems. But isn't this sort of state objectivism strictly inconsistent with state antirealism? If the quantum state is not real, then how could one be wrong about the quantum state? In order to answer this question, Healey engages in subtle reasoning about how objective correctness can be disentangled from the correspondence theory of truth, and about how the meaning of the quantum state can be accounted for in an inferential theory of content. This just goes to show that matters are not as simple as they initially appeared to be.

Healey's subtlety is laudable, but sometimes it verges on doublespeak. For example, Healey hangs much on the distinction between ascribing a state $\psi$ to a thing $X$, and describing $X$ with $\psi$.

Pragmatists agree with QBists [quantum Bayesians] that quantum theory should not be thought to offer a description or representation of physical reality: in particular, to ascribe a quantum state is not to describe physical reality. (Healey, 2016, emphasis added)

What are we supposed to be doing when we "ascribe" a quantum state? If $\psi$ has no representational role whatsoever, then why speak of "ascribing" it to a physical object or situation? Why not just speak of "using" the wavefunction - as one uses a computer or a hammer — to get a job done?

In the English language, the word "ascribe" involves a subject postulating a relation between two objects: $S$ ascribes $Y$ to $X$. More is true: in normal conversation, to ascribe $Y$ to $X$ involves judging that there is a pre-existing relation between $Y$ and $X$. For example, "He ascribed Jane's short temper to her upset stomach." In this way, ascribing is different than using: I can use $Y$ to do something to $X$ without making any judgment about the relation between $Y$ and $X$. These considerations show that the word "ascribe" is tantalizingly close to other words — such as "describe" — that connote the 
existence of a representational relation, exactly the sort of thing that Healey wishes to deny. To consistently carry out his pragmatist program, Healey should use a different word than "ascribe".

Here's what I think is really going on here. The phrase " $Y$ describes $X$ " is rather vague; and being vague, it can be thought to license all sorts of inferences about the relationship between $Y$ and $X$. When people say that " $Y$ describes $X$ ", they tend to import a lot of baggage that goes far beyond the simple existence of a representational relation between $Y$ and $X$. In fact, it seems all too easy to fall into the mistake of thinking that $Y$ describes $X$ only if $Y$ is similar to $X$. Of course that's not true: the phrase "is over six feet tall" describes Goliath, but this phrase is not similar to Goliath.

That temptation to assume similarity is all the more difficult to resist when the first argument of " $Y$ describes $X$ " is a geometrical object such as a wavefunction. The reason we fall into this trap, I assume, is because we do frequently use geometric objects as pictorial representations. For example, I might draw a rectangle on a piece of a paper and say, "this rectangle describes the shape of my desk." In this case, the rectangle on the paper is indeed similar to the desk in a well-defined mathematical sense.

Healey, Rovelli, and other self-proclaimed antirealists have surrendered too much to their opponents. They have allowed their opponents to define words like "ontological status" and "describes". Then, because Healey and Rovelli reject the implications that come along with this particular definition of "describes", they're forced to say that the quantum state does not de-

scribe at all. Thus, Healey and Rovelli lay themselves open to the charge of antirealism — which, of course, carries highly negative connotations. To be an antirealist implies a sort of failure of courage; it implies a sort of retreat. Ergo, Healey and Rovelli are seen as making less bold assertions about reality than their realist counterparts are making.

\section{The state as directly representing}

Are you a realist about the quantum state? We've already seen that this question cannot be paraphrased as, "Do you believe that the quantum state exists?" So what could the question mean? According to Wallace and Timpson,

Traditionally realist interpretations... read the quantum state literally, as itself standing directly for a part of the ontology of the 
theory. (Wallace and Timpson, 2010, p 703)

In fact, Wallace (2018b) locates the crucial divide between "representational" and "non-representational" views of the quantum state. Thus the shift is signaled from the material mode of speech (does the state exist?) to the formal mode of speech (does the state represent?). In particular, Wallace and Timpson claim that realism involves commitment to both literal and direct representation. Thus, Carroll utters the shibboleth when he says, "the wave function simply represents reality directly" (Carroll, 2017, 167). But what work is the word "directly" doing here? I'm led to think that the task of representing must be a bit like getting to work, where you have to take the right turns in order to follow the most direct route. So what are the instructions for following the direct route to representation?

When a person says that $Y$ represents $X$, then that typically signals that she endorses some inferences of the form:

(†) If $Y$ has property $\phi$ then $X$ has property $\phi^{\prime}$,

where $\phi \mapsto \phi^{\prime}$ is some particular association of properties (the details of which needn't detain us). Let's call $(\dagger)$ a property transfer rule. For example, if I say that a certain map represents Buenos Aires, then I mean that some facts about Buenos Aires can be inferred from facts about the map.

What then is the force of insisting that $Y$ doesn't merely represent $X$, but that it represents $X$ directly? I suspect that the word "directly" is supposed to signal endorsement of quite liberal use of property transfer rules. But just how liberal? The key question to keep in mind is: which specification of permitted property-transfer inferences corresponds most closely to the notion of "direct representation" that is favored by realists such as Carroll, Wallace, Saunders, and Timpson. When they say that " $\psi$ directly represents reality", what exactly are they saying about the relation between $\psi$ and the world?

Consider first the proposal:

(DR1) $Y$ directly represents $X$ just in case every property of $Y$ is also a property of $X$.

This proposal is logically consistent, but also absurd. One of the properties that $X$ has is being identical to $X$. Thus, according to DR1, if $Y$ directly represents $X$ then $Y=X$. Could Wallace and Timpson possible intend this? Does Carroll mean to say our universe is a subset of $\mathbb{R}^{3 n} \times \mathbb{C}$ ? If so, then 
scientific realism is truly a radical point of view. The wavefunction is an abstract mathematical object. Thus, if the universe is a wavefunction, then the universe is an abstract mathematical object. Perhaps mathematicians will applaud this conclusion, because then pure mathematics tells us everything there is to be known about the universe.

I suspect that the realists don't mean their direct representation claim in the sense of DR1. Let's try a more reasonable proposal.

(DR2) $Y$ directly represents $X$ just in case each mathematical property of $Y$ corresponds to some physical property of $X$.

Here we need some precise account of the "mathematical properties" of $Y$. According to standard set-theoretic foundations of mathematics, the mathematical properties of $Y$ are precisely those properties that can be described in the language of Zermelo-Fraenkel (ZF) set theory. Thus, for example, the mathematical properties of $Y$ would include its size (cardinality). In contrast, arbitrary predicates in natural language don't pick out mathematical properties of $Y$. For example, "is an abstract object" cannot be articulated in ZF set theory, and so wouldn't count as a mathematical property of $Y$. Thus, DR2 doesn't say that "anything goes" in terms of the representationally significant properties of $Y$.

Even so, DR2 is still implausibly profligate in the number of representationally significant properties it assigns to the wavefunction. In particular, for each definable name $c$ in ZF set theory, there is a definable predicate $\Theta_{c}$ given by

$$
\Theta_{c}(S) \leftrightarrow c \in S
$$

Among these definable set names, we have $\emptyset,\{\emptyset\}$, and so on. Now, a wavefunction is a function $\psi: A \rightarrow B$, with domain set $d_{0} f=A$ and codomain set $d_{1} f=B$. Thus, for any definable name $c$, it makes sense to ask whether $\Theta_{c}\left(d_{0} \psi\right)$, i.e. whether $c$ is contained in the domain of $\psi$.

Imagine now the following scenario. Two physicists, Jack and Jill, are arguing about whose wavefunction is a better representation of the universe. The funny thing is, Jack and Jill's wavefunctions are both Gaussians, centered on 0 , and with the same standard deviation. If you ask Jack to draw a picture of his wavefunction, then he draws a Gaussian centered at 0. If you ask Jill to draw a picture of her wavefunction, then she also draws a Gaussian centered at 0 . They agree that this picture is a correct representation of their respective wavefunctions. They also agree that their wavefunctions 
are written in the configuration space basis, and that the origin 0 represents the same point in the universe. It seems that there is nothing left for them to disagree about.

And yet, Jack and Jill insist on that their wavefunctions cannot both be correct. According to Jack, the correct wavefunction $\psi$ has the property that $\Theta_{\emptyset}\left(d_{0} \psi\right)$, that is, the empty set is an element of the domain of the wavefunction. According to Jill, the correct wavefunction $\psi^{\prime}$ does not have that property. They both believe that $\Theta_{\emptyset}$ corresponds to a genuine physical property. Jack asserts that this property is instantiated, and Jill asserts that it is not.

Jack and Jill would fail their quantum mechanics course. They don't understand how the theory works. In using the formalism of quantum theory to represent reality, we don't care about these fine-grained set theoretic differences. If two wavefunctions have the same shape, then we consider them to be the same. If two wavefunctions can be described via the same equation, then we take them to be identical. But what is this notion of same shape that we are using here? How can we tell when two wavefunctions are the same, at least for the purpose of doing physics?

At this point, we might want to lay down the ace card of recent philosophy of science: the notion of isomorphism. Can't we just say that two wavefunctions are representationally equivalent just in case they are isomorphic? In this case, we could then propose the following criterion for direct representation:

(DR3) $Y$ directly represents $X$ just in case $Y$ and $X$ are isomorphic.

This proposal sounds a lot more plausible than the previous two - especially because the word "isomorphism" is simultaneously precise (within certain fixed contexts), and flexible (since it means different things in different contexts). But that's precisely the problem with DR3: the phrase " $Y$ is isomorphic to $X$ " is no better defined than the phrase " $Y$ directly represents $X^{\prime \prime}$.

In mathematics, isomorphism is a category-relative concept. If you hand me two mathematical objects and ask, "are they isomorphic?" then I should reply by asking "which category do they belong to"? For example, two mathematical objects can be isomorphic qua groups, but non-isomorphic qua topological spaces. Thus, it makes no sense to say that a mathematical object is isomorphic to the world tout court. In order to make sense, we would first 
have to specify a relevant type (or category) of mathematical objects. For example, one might say that the world is isomorphic to a topological space $Y$, as shorthand for saying that the world has topological structure, and is in this sense isomorphic to $Y$. But if you give me a concrete mathematical object $A$ and say that the world is isomorphic to $A$, then I have no idea what you are saying.

So, if we want to say that the world is isomorphic to a wavefunction $\psi$, then we need to say what category of mathematical objects we take $\psi$ to belong to. And that's not going to be easy, for $\psi$ is not a group, or a topological space, or a differentiable manifold, or any other of the standard types of mathematical structure. There is no category of wavefunctions; and there is no notion of isomorphism between wavefunctions. ${ }^{4}$ The closest we come to finding a home for $\psi$ is in the category of Hilbert spaces: $\psi$ is an element of a Hilbert space, which is an object in the category of Hilbert spaces. But that won't help, because we don't want to say that the world is isomorphic to the Hilbert space $H$, but that it's isomorphic to a particular wavefunction $\psi$.

There are numerous other problems with analyses of representation in terms of isomorphism, some of which are discussed in (Frigg and Nguyen, 2016). We mention two further problems here, each of which might be taken to deliver a fatal blow to the account. First, an isomorphism is a function between two mathematical objects, and the world is not a mathematical object. In fact, as pointed out long ago by Reichenbach (1965), the only grip we have on the structure of the world is by means of our representations.

Second, our account of representational significance should mesh with our account of theoretical equivalence, and many philosophers of science hold views of theoretical equivalence according to which equivalent theories need not have isomorphic models. For example, Halvorson (2012) labels this view as "the model isomorphism criterion of theoretical equivalence", and he argues that it must be rejected. However, if the model isomorphism criterion of theoretical equivalence is rejected, then we must also reject the claim that representation entails isomorphism between the world and one of the theory's models. We can argue as follows: if two theories $T$ and $T^{\prime}$ are equivalent, and if $T$ is representationally adequate, then $T^{\prime}$ is also representationally

\footnotetext{
${ }^{4}$ It won't help to say that $\psi$ and $\psi^{\prime}$ are isomorphic wavefunctions just in case there is a unitary symmetry $U$ such that $U \psi=\psi^{\prime}$. For in that case, all wavefunctions would be isomorphic.
} 
adequate. But if the models of $T$ are not isomorphic to the models of $T^{\prime}$, then it cannot be the case that the world is isomorphic to a model of $T$ and also to a model of $T^{\prime}$. Therefore, to say that $T$ is representationally adequate does not entail that the world is isomorphic to one of the models of $T$.

\section{Representationally significant properties}

As we have seen, isomorphism-based analyses of representation have difficulty explaining how wavefunctions represent - because there is no obvious candidate notion of "isomorphism" for wavefunctions. Perhaps, however, we can attack this problem from the other side. Having a notion of isomorphism in place gives us a criterion for identifying representationally significant properties:

A property $\phi$ is representationally significant just in case $\phi$ is invariant under isomorphism.

But of course, we needn't already have a notion of isomorphism in place to choose the representationally significant properties. We can simply say what those properties are.

As we know, it would be disastrous to propose that all mathematical properties of a wavefunction are representationally significant. For example, the property of "having domain that contains the element $\{\emptyset,\{\emptyset\}\}$ " is a perfectly good mathematical property that a wavefunction either possesses or does not possess. But nobody, to my knowledge, has ever proposed that this mathematical property represents a bona fide physical property. In practice, we simply don't care whether we use a wavefunction $\psi$ that has that property, or a similar wavefunction $\psi^{\prime}$ that lacks that property. Many of these set-theoretically definable properties of a wavefunction are routinely ignored as "surplus mathematical structure".

In my experience, physicists can't usually say explicitly which properties of $\psi$ are representationally significant. However, we can determine which properties of $\psi$ they care about by watching what they do. If they treat two wavefunctions $\psi$ and $\psi^{\prime}$ as interchangeable, then their behavior suggests that they accord no representational significance to properties that separate these

two functions. Here we say that a property $\Theta$ separates $\psi$ and $\psi^{\prime}$ just in case $\Theta(\psi)$ and $\neg \Theta\left(\psi^{\prime}\right)$. 
The art of discriminating between wavefunctions is not so unlike the fabled art of "chicken sexing". The skilled chicken-sexer has the ability to judge reliably whether two chicks are of the same sex. But is you ask him what criteria he is using, he will be at a loss for words. In the same way, the skilled quantum mechanic has the ability to judge whether two wavefunctions are representationally equivalent. And she displays her judgment of representational equivalence by her disinterest in the question, "which of these two wavefunctions provides the correct representation of reality?"

I'm not sure that it would be possible to give a fully explicit account of the equivalence relation of "representational equivalence" for wavefunctions. Nonetheless, there are certain sufficient conditions for representational equivalence that are uncontroversial.

First, two wavefunctions are representationally equivalent if one is a complex multiple of the other - i.e. if they lie in the same ray in Hilbert space. Thus, if a property $\Theta$ of wavefunctions is not invariant under this relation, of lying in the same ray, then $\Theta$ is not representationally significant. For example, consider the property $\Theta$ given by

$$
\Theta(\psi) \leftrightarrow(\psi(0)=1)
$$

Clearly there are two functions $\psi$ and $\psi^{\prime}$ such that $\psi \sim \psi^{\prime}$, but $\Theta(\psi)$ and $\neg \Theta\left(\psi^{\prime}\right)$. Therefore, $\Theta$ is not a representationally significant property of wavefunctions.

Second, wavefunctions are not actually functions at all. In fact, the space of square integrable functions on configuration space is not a Hilbert space. Instead, to define a positive-definite inner product, one has to take equivalence classes of functions relative to the equivalence relation $\sim$ of "agreeing except on a set of measure zero." But now consider the property $\Theta$ defined by:

$$
\Theta(\psi) \leftrightarrow\left(|\psi(0)|^{2}=1\right)
$$

Again, there are two functions $\psi$ and $\psi^{\prime}$ such that $\psi \sim \psi^{\prime}$, but $\Theta(\psi)$ and $\neg \Theta\left(\psi^{\prime}\right)$. Therefore, $\Theta$ is not a representationally significant property of wavefunctions.

This is not to say that there are no representationally significant properties of wavefunctions. For example, consider the property

$$
\Theta(\psi) \leftrightarrow \int_{\Delta}|\psi(x)| d \mu(x)=1 .
$$


This property $\Theta$ can be shown to be invariant under the equivalence relations above. Indeed, practitioners of quantum theory know exactly what this property is: it's the property $[Q \in \Delta]$ of being located in the region $\Delta$. What other invariant properties are there? Can we give some sort of systematic description of them?

As mentioned before, the Hilbert space formalism is normally taken to represent properties by means of the subspaces of the statespace. Let's think about how this works in the case of the space $L_{2}(X)$ of (equivalence classes of) wavefunctions. What does a subspace of $L_{2}(X)$ look like? Some subspaces correspond to properties of functions. For example, consider the property

$$
\Theta(\psi) \equiv \psi \text { has support in the region } \Delta .
$$

It's not difficult to see that the set of functions satisfying $\Theta$ forms a closed subspace of $L_{2}(X)$. But not every subspace of $L_{2}(X)$ has such an interpretation in terms of straightforwardly geometric features of functions. For example, let $U: L_{2}(K) \rightarrow L_{2}(X)$ be the unitary isomorphism between the momentum-space and position-space representation of wavefunctions. Now begin by defining the same sort of subspace, but relative to the momentumspace representation. That is, let $E$ be the subspace of $L_{2}(K)$ consisting of functions with support in $\Delta$. The natural interpretation of $E$ is: having momentum value in the set $\Delta$. Then $U(E)$ is a subspace of $L_{2}(X)$, and hence represents a quantum-theoretic property $\Theta$. But this property $\Theta$ doesn't manifest itself as a natural property of functions on the original configuration space $X$. Indeed, it's not clear that it would be possible to express $\Theta$ without making reference to the isomorphism between $L_{2}(K)$ and $L_{2}(X)$.

We have here a nice concrete example of an issue that philosophers have been discussing in the abstract - the issue of abundant versus sparse views of properties (see Bricker, 1996). The Hilbert space formalism gives a special version of the sparse view of properties: not every subset of $L_{2}(X)$ corresponds to a natural property. One might think initially that this sparse view makes life difficult by preventing us from saying certain things. For example, as Wallace (2012) points out, this sparse view entails that "has a definite value of energy" fails to pick out a property (a consequence which he finds to be unacceptable).

However, there is an obvious problem with trying to take an abundant view of the properties of quantum theory, i.e. with taking every subset of $L_{2}(X)$ to pick out a physical property. The problem is that there are too many such subsets, and their physical interpretation is unclear. Nonetheless, 
the Hilbert space formalism provides a method for identifying those subsets of $L_{2}(X)$ that represent physical properties. In particular, we have the following result:

(SQ) Let $H$ be an abstract Hilbert space of countably infinite dimension. Then each subspace of $H$ is of the form $U^{-1}[Z \in \Delta]$, where $U: H \rightarrow L_{2}(\mathbb{R})$ is a unitary isomorphism, $\Delta$ is a Borel subset of $\mathbb{R}$, and $[Z \in \Delta]$ is the subspace of functions with support in $\Delta .^{5}$

Here we think of $L_{2}(\mathbb{R})$ as wavefunctions of some particular dynamical variable $Z$, which could be position (along some axis), or momentum (along some axis), or energy, or .... In this case, $U^{-1}[Z \in \Delta]$ is the subspace of wavefunctions where the value of $Z$ lies in $\Delta$. In other words, $U^{-1}[Z \in \Delta]$ and $[Z \in \Delta]$ represent the same property — only, this property's physical interpretation is more perspicuous in the latter case.

Thus, there is a non-trivial question about which properties of functions (i.e. subsets of $\left.L_{2}(X)\right)$ represent bona fide, or "natural", physical properties. Take an arbitrary mathematical predicate of functions, such as

$$
\Theta(\psi) \equiv \psi \text { is a smooth (i.e. infinitely differentiable) function, }
$$

which seems to be quite natural, at least from a mathematical point of view. But why suppose that $\Theta$ represents a natural physical property? What criteria should we use to sort out the genuine predicates from the spurious predicates? Some might suggest an operationalist criterion:

(operationalist) A predicate $\Theta$ of wavefunctions represents a natural physical property iff there is a measurement that would verify whether an object's state $\psi$ has property $\Theta$.

But that criterion is too imprecise. And, in any case, the operationalist criterion is stricter than quantum theory's own criterion, which countenances many natural properties that cannot be operationally detected.

The language of quantum theory, represented via the Hilbert space formalism, comes with a vocabulary, including a list of predicates.

\footnotetext{
${ }^{5}$ This result is part of the folklore of functional analysis, and may be reconstructed from the results in Chapter 9 of (Kadison and Ringrose, 1991).
} 
(QM properties) A predicate $\Theta$ of wavefunctions represents a natural physical property iff the set $\left\{\psi \in L_{2}(X) \mid \Theta(\psi)\right\}$ is a subspace of $L_{2}(X)$.

By the result above, this criterion can be restated as follows:

(QM properties) A predicate $\Theta$ of wavefunctions represents a natural physical property iff there is a dynamical variable $Z$, and a measurable $\Delta \subseteq \mathbb{R}$, such that $\Theta(\psi)$ if and only if $\psi$ lies in the subspace $[Z \in \Delta]$.

These predicates can then be taken as giving quantum theory's preferred account of natural properties. In short, the natural properties are precisely those picked out by saying that a quantity's $Z$ has value in a certain range.

So, we return to the original question: if $\Theta(\psi)$ is the predicate " $\psi$ is a smooth function", then does $\Theta$ pick out a physical property of wavefunctions? Quantum theory answers this question by saying: $\Theta$ represents a physical property only if there is some quantity $Z$ such that that $\Theta$ picks out the subspace $[Z \in \Delta]$.

When we talk about giving a "physical interpretation" to a subset $E$ of statespace, the demand is not that $E$ be given an operational interpretation, as, e.g., corresponding to some measurement operation. Instead, we're simply asking that the mathematical object $E$ be describable in words that have some antecedent physical meaning. It's simply the demand that we understand what the formalism purports to represent.

\section{$5 \quad$ Reading the state literally}

Recall that Wallace and Timpson say that a quantum state realist does two things: (1) she believes that the state represents reality directly, and (2) she reads the state literally. As we saw, there are various ways of cashing out " $Y$ directly represents $X$." If you push the notion to the extreme, where $Y=X$, you'll end up saying stupid things. But as soon as you start to nuance this notion, you start to sound less like a full blooded realist.

So can we find a firm foothold for realism in the second criterion? Is it the commitment to a "literal reading of the state" that sets the quantum state realists apart from their antirealist counterparts? Here we've tapped into a central vein in philosophical discussions of scientific realism. For example, 
forty odd years ago, van Fraassen described scientific realism as the belief that:

The aim of science is to give us a literally true story of what the world is like; and the proper form of acceptance of a theory is to believe that it is true. (Van Fraassen, 1976, emphasis added)

The debates of the last forty years seem not to have brought into question the connection between realism and literalism. In a recent authoritative account of scientific realism, Chakravartty reasserts the connection:

Semantically, realism is committed to a literal interpretation of scientific claims about the world. (Chakravartty, 2017, emphasis added)

But something fishy must be going on here. The idea that a scientific theory is a set of claims (i.e. sentences) fell out of favor about forty years ago. Nowadays, most philosophers of science say that a scientific theory consists of a collection of models, plus some claim to the effect that one of these models represents the world. But if a theory is a collection of models, then how am I supposed to read it literally? Nor can this problem be brushed away by adopting a different view of scientific theories. For better or worse, the theories of mathematical physics involve collections of mathematical models, such as Lorentzian manifolds, Hilbert spaces, etc.. So how then are we supposed to read these theories literally?

The answer, in short, seems to be: to read a theory literally is to take one of its models $M$ as a reliable guide to features of the world. But now we are right back to where we were when considering analyses of " $Y$ directly represents $X$ ". If I'm a literalist about $M$, then which features of $M$ should I take to be representationally significant? The simple answer "all features of $M$ " leads immediately to absurdity. The answer "all mathematical features of $M$ " also leads to a bizarre and untenable picture. Thus, we're thrown back on a more piecemeal approach, where one has to know how to interpret the model $M$, which means being able to distinguish its representationally significant properties from the insignificant ones.

Indeed, learning how to use a physical theory requires that learning the art of "reading claims off" of a model. Consider, for example, the General Theory of Relativity (GTR), where a model $M$ is a Lorentzian manifold. What might it look like to read $M$ literally? Well, GTR claims that at each 
point $p \in M$, there is a four-dimensional tangent space $T_{p}$. And living on top of $T_{p}$ there is an infinite tower of $(m, n)$ tensors, for all natural numbers $m$ and $n$. Are these things I've just said among the "scientific claims" of GTR? If I'm a realist about GTR, then am I committed to these claims? Should I envision an infinitely extended tangent space $T_{p}$ of four dimensions sitting on the tip of my nose, and indeed, a different such tangent space for each instant of time? Are these tangent spaces "part of the furniture of the world". If this is what it means to be a realist about GTR, then Einstein was no realist.

To make the point more clearly, GTR entails that:

For each point $p \in M$, there is an open neighborhood $O$ of $p$, and a coordinate chart $\phi: O \rightarrow \mathbb{R}^{4}$.

These coordinate charts are just as much elements of a model of GTR as a wavefunction is an element of a model of QM. Thus, if literalism demands commitment to the wavefunction $\psi$, then it also demands commitment to the coordinate chart $\phi$. If quantum state realism is just a "literal reading of QM", then coordinate chart realism is just a "literal reading of GTR".

If you don't think that GTR involves a commitment to an ontology of tangent spaces, coordinate charts, etc., then I can only agree: not every true statement, made within the language of a theory, is one of the "scientific claims" of that theory. To say that a model $M$ accurately represents the physical world does not mean that every mathematical thing in $M$ represents a physical thing. Realism, according to Chakravartty, Timpson, Wallace, van Fraassen, et al., requires commitment to the scientific claims of a theory, interpreted literally. But you can't interpret a mathematical object literally. That simply doesn't make sense. The demand for literal interpretation only makes sense after we have used the formalism to express claims in a language that we understand.

Here we have to lay some blame at the door of the semantic view of theories. The semantic view of theories plus realism suggests the idea that one ought to interpret models literally — an idea that can lead to absurd consequences if not further nuanced. A model's elements need not all play the same representational role. For example, suppose that I make a map of Princeton University, on which I draw several buildings. Suppose that I also draw a picture of a compass in the lower right hand corner of my map - to indicate its orientation. Now, I'm a realist about the geography of Princeton, and I believe that my map is a faithful representation of it. But that doesn't 
mean that I believe there is a huge compass lying on the ground just outside of the university. Nor would I say that the compass on the map is "just a bookkeeping device", or that it "has no representational role". The compass does have a representational role: it represents a claim about how my map is related to the actual town of Princeton. And if this compass can be said to have a representational role, then so can a wavefunction. ${ }^{6}$

\section{Spacetime state realism}

The most recent development in the realist ontology program is the proposal to upgrade wavefunction realism to "spacetime state realism" (see Wallace and Timpson, 2010; Wallace, 2018a). But does this technical maneuver dodge the various philosophical problems that confront wavefunction realism? In order to press the question further, we need to sketch the idea behind spacetime state realism.

Let's begin with the simplest (and least interesting) case of spacetime state realism - the case where spacetime consists of a single point. In this case, we represent a quantum system by means of a $C^{*}$-algebra $\mathcal{A}$ of observables. [For an account of this formalism, see (Ruetsche, 2011).] The important point is that $\mathcal{A}$ is closed under operations of addition, multiplication, and conjugation $A \mapsto A^{*}$. Moreover, there is a preferred multiplicative unit $I \in \mathcal{A}$, the identity operator. The prototypical case of a $C^{*}$-algebra is the algebra of $n \times n$ complex matrices.

We need a few definitions: an operator $A \in \mathcal{A}$ is said to be self-adjoint just in case $A^{*}=A$, and $A$ is said to be positive just in case $A=B^{*} B$ for some operator $B \in \mathcal{A}$. A function $\omega: \mathcal{A} \rightarrow \mathbb{C}$ is said to be a linear functional just in case $\omega(c A+B)=c \omega(A)+\omega(B)$ for all $A, B \in \mathcal{A}$ and $c \in \mathbb{C}$. A linear functional $\omega$ is said to be positive just in case $\omega(A) \geq 0$ for every positive operator $A \in \mathcal{A}$. A positive linear functional $\omega$ is said to be a state just in case $\omega(I)=1$. We will use $\Sigma(\mathcal{A})$ to denote the space of states of $\mathcal{A}{ }^{7}$

We can formulate quantum mechanics in the language of $C^{*}$-algebras just as well as we can in the language of Hilbert spaces. Indeed, the self-adjoint operators in $\mathcal{A}$ represent observables (or more accurately, quantities), and the

\footnotetext{
${ }^{6}$ For an illuminating investigation of the notion of "literal interpretation", see (Hirsch, 2017).

${ }^{7}$ Although it won't be important for our considerations here, $\Sigma(\mathcal{A})$ has quite a bit of interesting structure, as described, e.g., in (Alfsen and Shultz, 2012).
} 
elements of $\Sigma(\mathcal{A})$ represent physical states. As a particular case in point, if $\mathcal{A}$ is the algebra of $2 \times 2$ matrices, then the self-adjoint operators are simply the Hermitian matrices, and the states on $\mathcal{A}$ correspond one-to-one with density operators on $\mathbb{C}^{2}$ via the equation

$$
\omega(A)=\operatorname{Tr}\left(W_{\omega} A\right) .
$$

With these definitions in hand, we can state Wallace and Timpson's proposal quite simply:

For a system represented by the algebra $\mathcal{A}$, the properties correspond one-to-one with the states in $\Sigma(\mathcal{A})$.

This proposal can be made more picturesque and plausible if you think of a "field of states", where each point $p$ in spacetime is assigned a state $\omega_{p}$. And if you feel that this is just empty mathematics, then it might help to think of the typical case, where $\omega_{p}$ is represented concretely by a density operator $W_{p}$. Then the field $p \mapsto W_{p}$ of density operators starts to look more like a classical field configuration, where some mathematical object, such as a tensor, is assigned to each point in space. The only mathematical difference is that $W_{p}$ is a complex matrix instead of a tensor. But as Wallace and Timpson point out, the relative unfamiliarity of complex matrices such as $W_{p}$ shouldn't rule them out as legitimate values of a physical field.

To this point, I agree with Wallace and Timpson. What bothers me is not the difference between tensors and complex matrices. What bothers me is the conflation of the various theoretical roles of states, quantities, and properties. The typical job of states is to assign values to quantities. So, if we ask states also to serve as values of quantities, then the the job of states will be to assign states. In order to try to keep things straight in our heads, we might try to declare some "types". First, the standard way of thinking of states is that they are of type $\mathbf{Q} \rightarrow \mathbf{V}$, where $\mathbf{Q}$ is the quantity type, and $\mathbf{V}$ is the value type. But now, Wallace and Timpson tell us that states are also of type $\mathbf{V}$. In this case, states would be both of type $\mathbf{Q} \rightarrow \mathbf{V}$ and of type $\mathbf{V}$, resulting in a type confusion.

What's more, we typically ask a physical theory to provide some sort of "state-to-property" link. For example, the so-called orthodox interpretation of quantum theory proposes the eigenstate-eigenvalue link:

(EE link) A property $E$ of the system is possessed in state $\psi$ just in case $E \psi=\psi$. 
Wallace and Timpson also propose a state-to-property link. However, their properties are of the form "being in state $W$ ", and so their proposal reduces to:

(WT link) A system has property $W$ when it is in state $W$

Or perhaps it would be better to say:

(WT link) A system has the property of being in state $W$ just in case it is in state $W$.

I suppose this claim is true. But I didn't need to learn any physics to draw that conclusion. This is nothing more than a disquotational theory of truth.

It's possible that Wallace and Timpson's proposal only trivializes in the trivial case — where spacetime consists of a single point. Perhaps their proposal is only meant to give an interesting picture in the case where we associate a different algebra of observables $\mathcal{A}(O)$ to each region $O$ of spacetime. In that case, their recipe would yield a much richer structure, something like a co-presheaf of states (see Swanson, 2018). But I don't see any reason to think that this additional mathematical structure can undo the conflation of states and properties that already occurs at the level of individual algebras.

Finally, even if you can get past these other worries, there is a worry that the Wallace-Timpson proposal shows too much. Indeed, there's a case to be made that any reasonable generalized probability theory can be formulated in the framework of $C^{*}$-algebras. In that case, it would seem that the Wallace-Timpson proposal yields a realistic physical ontology for any reasonable generalized probability theory. In other words, it's realism on the cheap.

\section{The wavefunction as symbol}

We began our discussion with the dilemma: either the quantum state has ontological status, or it does not. We saw that this dilemma cannot be taken seriously, because a state isn't a candidate for existence or non-existence in the physical sense. Thus the original ontological dilemma was transformed into a representational one: either the quantum state represents reality, or it does not. But then we discovered that "represents" can be understood in many different senses - and in the most extremely realistic sense of "represents", no sane person would say that the quantum state represents the 
world. Thus, the disagreement between realists and antirealists - where it is not simply a matter of emotional associations with words - boils down to different stories about how to use the quantum state to represent reality.

It's ironic, then, that early interpreters of quantum theory — such as Bohr and Carnap - are often assumed to be operationalists about the quantum state. That couldn't be further from the truth. Both Bohr and Carnap explicitly say that the wavefunction is not merely a calculational device. Presumably, somebody ran a word-search on Bohr and Carnap's writings, and having found no hits for " $\psi$ represents reality directly" or " $\psi$ has ontological status", they concluded that these guys must have been antirealists.

There is another possibility that we should take seriously. What if Bohr and Carnap intentionally exercise caution with words like ontological status and direct representation, since those words might lead to a misunderstanding of how quantum theory works. Perhaps Bohr and Carnap were groping their way, if ever so haltingly, toward a more articulate account of how the wavefunction represents reality.

\subsection{Bohr}

Analytic philosophers have been quick to categorize Bohr as an operationalist about the wavefunction, citing statements like this one:

...the symbolic aspect of Schrödinger's wave functions appears immediately from the use of a multidimensional coordinate space, essential for their representation in the case of atomic systems with several electrons.

Faye (2014), for example, seems to think that Bohr's use of "symbolic" is code for "should not be taken literally".

Thus [for Bohr], the state vector is symbolic. Here "symbolic" means that the state vector's representational function should not be taken literally but be considered a tool for the calculation of probabilities of observables.

Faye's confusion here is understandable. We analytic philosophers of science tend to associate "symbolic" with "non-referential" or "uninterpreted." In particular, with regard to a sentence $X$ in a formal calculus, to say that " $X$ is symbolic" means precisely that $X$ in uninterpreted, and so lacks a truth 
value. In other words, when we hear "symbol", we immediately think "does not purport to describe reality".

However, the considerations of previous sections show that this usage of "symbolic" doesn't make much sense when $X$ is a mathematical object, such as a wavefunction. Nor would it make much sense to attribute this usage of "symbolic" to Bohr, who didn't use words in exactly the way that analytic philosophers of science have come to use them. When Bohr uses "symbolic", I assume that his meaning draws on a his peculiar educational background, which was heavy on continental figures such as Kant, Goethe, Hegel, and Helmholtz. I assume that his meaning was also shaped by his interactions with continental-type philosophers such as Ernst Cassirer, and mathematicians such as his brother Harald. Thus, when Bohr says something is "symbolic", we shouldn't immediately conclude that he means it in the sense of the uninterpreted predicate calculus.

Indeed, one of Bohr's students, Christian Møller, asked him explicitly what he meant by calling the wavefunction "symbolic". In a 1928 letter, Bohr replies in so many (!) words:

Regarding the question discussed in your letter about what was meant, when I in my article in Naturwissenschaften, emphasized so strongly the quantum-theoretical method's symbolic character, I am naturally in complete agreement with you that every description of natural phenomena must be based on symbols. I merely sought to emphasize the fact, that this circumstance that in quantum theory, we typically use the same symbols we use in the classical theory — doesn't justify our ignoring the large difference between these theories, and in particular necessitates the greatest caution in the use of the intuitive concepts [anskuelsformer] to which the classical symbols are connected. Naturally, one doesn't easily run this danger with the matrix formulation, where the calculation rules, which diverge so greatly from the previously standard algebraic ones, hold quantum theory's special nature before our eyes. Furthermore, to use the word "symbolic" for non-commutative algebra is a way of speaking that goes back long before quantum theory, and which has entered into standard mathematical terminology. When one thinks about the wave theory, it is precisely its "visualizability" [anskuelighed] which is simultaneously its strength and its snare, and here by emphasizing 
the approach's [behandlingens] symbolic character, I was trying to bring to mind the differences - required by the quantum postulate - from classical theories, which are hardly ever sufficiently heeded. (Bohr, 1928, original in Danish)

As is typical with reading Bohr, one doesn't feel that the situation has been greatly clarified. However, one thing is clear: Bohr does not intend to single out the quantum state for operational treatment. If Bohr is an antirealist about the quantum state, then he is an antirealist about all of mathematical physics. For Bohr, all mathematical representation is "symbolic", whether observable or unobservable aspects of reality are being represented. Among the symbolic representations of physics, he would include the $F_{a b}$ of Maxwell's equations, the $g_{a b}$ of General Relativity, as well as functions representing the trajectories of material bodies through spacetime. Bohr's point might be summed up simply by saying that mathematical objects are not sentences, and so they cannot "be read literally".

To understand Bohr's use of "symbolic", it might also help to look at a philosopher whose career ran in parallel with his. In fact, it's well known that Bohr interacted extensively with Ernst Cassirer when the latter was composing his book Determinismus und Indeterminismus in der Modernen Physik, first published in 1937. Whether there is a more substantial overlap in their usage of "symbolic" will have to await more detailed historical investigations.

Nonetheless, it is clear that there are many common themes in the views of Bohr and Cassirer (see e.g. Pringe, 2014). One such common theme is giving careful thought to the way that mathematical objects can be used to represent the physical world. In putting forward his views on this issue, Cassirer is clear that "symbolic" should not be opposed to "representational". The interesting question is not whether something is representational, but rather how it represents. In particular, Cassirer believes that the development of mathematics and physics in the 19th century provides a particularly clear demonstration of the need to expand the notion of representation beyond a simplistic "similarity of content" account.

Mathematicians and physicists were first to gain a clear awareness of this symbolic character of their basic implements. ... In place of the vague demand for a similarity of content between image and thing, we now find expressed a highly complex logical relation, a 
general intellectual condition, which the basic concepts of physical knowledge must satisfy. (Cassirer, 1955, p 75)

For the former, more narrow, use of symbols, Cassirer uses the word Darstellingsfunktion. For the latter, more general, use of symbols, Cassirer uses the word Bedeutungsfunktion. Thus, to relate back to our earlier analysis of " $Y$ represents $X$ ", we might think that Darstellungsfunktion picks out a kind of representational relation that licenses many inferences about $X$ from $Y$, especially inferences having to do with spatiotemporal properties. The paradigm case, of course, of such representations are the directly geometric. In contrast, Bedeutungsfunktion picks out a more general kind of representation relation which does not imply geometric similarity between $X$ and $Y$.

Bohr does not avail himself of Cassirer's classification of symbolic forms. However, he does often speak of things being "unvisualizable" (uanskuelig) - opening a door to the deep dark recesses of the Kantian tradition. Bohr's notion of representing something to visual intuition doubtless overlaps in important ways with Cassirer's notion of Darstellungsfunktion. And if there is any coherence in Cassirer's idea of moving toward Bedeutungsfunktion, then Bohr may be blazing the same trail. In particular, when Bohr says that subatomic processes cannot be vizualized, he shouldn't be taken as saying that quantum theory is non-representational. Instead, Bohr might be groping his way toward a more nuanced account of how mathematics can be used to represent physical reality.

\subsection{Carnap}

We began the chapter with a story about how the early interpreters of quantum theory were operationalists. That story is often neatly combined with another story that post-Quinean analytic philosophers love to tell: the story about how silly and stupid the logical positivists were. According to this story, the logical positivists viewed scientific theories as "mere calculi" for deriving predictions. Thus, the story concludes, it's no surprise that Bohr et al. were operationalists about the quantum state, given that operationalism had so thoroughly infected the prevailing view of scientific theories.

If you've ever read a serious historical account of the origins of quantum theory, you know that the first story is mostly propaganda. None of the pioneers of quantum theory — Bohr, Heisenberg, Dirac, etc. — was a crass

operationalist. And if you've ever read a serious historical account of 20th 
century philosophy, you also know that the second story is largely Quinean propaganda. In fact, Carnap himself was a vocal critic of operationalism long before he felt the pressure of Quine's critiques of the positivist program.

Some, especially philosophers, go so far as even to contend that these modern theories, since they are not intuitively understandable, are not at all theories about nature but "mere formalistic constructions", "mere calculi". But this is a fundamental misunderstanding of the function of a physical theory. (Carnap, 1939, p 210)

Notice how Carnap feels the same pressure that Bohr and Cassirer feel the pressure that the new theories of physics are not "intuitively understandable". Moreover, like Bohr and Cassirer, he refuses to take the breakdown of intuitive understandability (or anskuelighed, or Darstellbarkeit) to demand a retreat to operationalism. Instead, Carnap - like Bohr and Cassirer asks us to think harder about how our theories purport to represent physical reality.

Like Bohr, Carnap also insists that the representational status of the quantum wavefunction is not all that different from the situation of the symbols of classical mathematical physics.

If we demand from a modern physicist an answer to the question what he means by the symbol " $\psi$ " of his calculus, and are astonished that he cannot give an answer, we ought to realize that the situation was already the same in classical physics. There the physicist could not tell us what he meant by the symbol " $E$ " in Maxwell's equations. ... Thus the physicist, although he cannot give us a translation into everyday language, understands the symbol " $\psi$ " and the laws of quantum mechanics. He possesses the kind of understanding which alone is essential in the field of knowledge and science. (Carnap, 1939, pp 210-211)

Interestingly, the words of Carnap here are echoed - quite unintentionally, I'm sure - by Wallace and Timpson.

... it's not as if we really have an intuitive grasp of what an electric or magnetic field is, other than indirectly and by means of instrumental considerations. ... Thus, it seems that we gain a basic understanding of the electromagnetic field by seeing it as a 
property of spatial regions, and our further understanding must be mediated by reflecting on its role in the theory. ... beyond that there doesn't seem to be much further to be grasped. (Wallace and Timpson, 2010, p 700)

We might just add that the concept of spatial regions doesn't provide us with a truly Archimedian reference point - for these regions themselves are understood in a mediated way, via their description in physical theory.

At this point, it should be thoroughly unclear how the views of Bohr, Cassirer, and Carnap differ from some of the more moderate and reasonable quantum state realists. To one such view we now turn.

\subsection{The nomological view}

According to the cutting edge survey of Chen (2018), there are three versions of wavefunction realism - the two $\psi$-field views, and a "nomological view" where the wavefunction represents a law of nature (Goldstein and Zanghì, 2013; Esfeld, 2014; Miller, 2014; Callender, 2015; Esfeld and Deckert, 2017). Thus, if we were to regiment the nomological view, we might say that the wavefunction plays the theoretical role of a proposition, or perhaps of a rule for generating propositions. The theoretical role of propositions is, of course, quite different than the theoretical role of names or even variables, both of which are used to denote existing things. Thus, only by stretching the word "ontological" beyond the breaking point could we say that the nomological view is ontological. No matter what view of laws we take, a law isn't a thing, and is not in the domain of quantification of a physical theory. Thus, according to the nomological view, the wavefunction is not a beable.

Why then should the nomological view of the wavefunction be called a realist view it doesn't treat the wavefunction as corresponding to an existing thing? Presumably, nomologists would say that what makes their view realist is that the propositions encoded in $\psi$ are objectively true, i.e. they correspond to reality. But what then are these propositions that are encoded in $\psi$ ? Of course, Bohmians have an answer ready at hand: $\psi$ encodes propositions about the trajectories of particles.

Notice that the specific Bohmian answer is not implicit in the very idea that $\psi$ encodes true propositions. Even a rank operationalist will say that $\psi$ encodes true propositions - about the probabilities of measurement outcomes. Only we might question whether these propositions are "objectively 
true", since probabilities of measurement outcomes are indexed by measurements, and the latter has yet to be objectively defined.

So what makes the nomological view realist? Is it simply that $\psi$ encodes objectively true propositions? Or is it that $\psi$ encodes true propositions about particle trajectories? I would be loath to accept the second answer, because it would make realism hostage to one idiosyncratic ontological picture, viz./ a particle ontology. Surely one can be a realist and have some sort of gunky ontology, or a field ontology. So, it seems that realist-making feature of the nomological view is merely its commitment to the idea that $\psi$ represents objectively real features of the world. But now, if that's enough to make

a view realist, then Healey's view is also a realist view. For Healey says that each physical situation is correctly represented by at most one quantum state. Healey and the nomologists agree that $\psi$ represents objectively real features of the world.

Nor can we say that the nomological view is more realist than Healey's because it takes $\psi$ to be a direct representation of reality. The representation relation posited by the nomological view is every bit as indirect and nuanced as that posited by Healey, or by Bohr for that matter. Indeed, the nomological view includes an intricate translation scheme from mathematical properties of $\psi$ to various meaningful physical statements, some of which are about occurent states of affairs, and some of which are about how things will change as time progresses. Thus, in terms of how $\psi$ represents, the nomological view is closer to the views of Healey, Bohr, and Carnap than it is to $\psi$-field views. The nomologists may be horrified to hear this, for they take great pride in being realists. But recall that Bohm often emphasized that his point of view was not so radically different from Bohr's. He even offered his point of view as a clarification of Bohr's. Perhaps then the nomological view could be thought of as an attempt to clearly articulate some of the things that Bohr was trying to say about the wavefunction.

\section{Conclusion}

The primary aim of this chapter was to investigate the meaning of realism about quantum theory, and in particular, realism about the quantum state. We found that, for the most part, these phrases are empty of substantive content. They are emotive catch phrases that are meant to muster the troops, and perhaps to sell books. But please don't get me wrong. I'm not saying 
that there are no substantive questions about how to interpret the quantum state. First of all, dissolving the anti/realism distinction doesn't solve the measurement problem. There's still the thorny issue of why it appears to us that measurements have outcomes. Second, there are genuine disagreements about how to use quantum states - even if these disagreements don't correlate directly with a distinction between "real" and "not real".

First, there is a genuine question of how to think of the relation of quantum states to physical situations. (For simplicity, I'll suppose that a physical situation is picked out by an ordinary language description, for example, by the sorts of instructions that one might give to an engineer or to a postdoc in the lab.) At one extreme, we have objectivists who think that each such situation corresponds to a unique correct quantum state. At the opposite extreme, we have the Quantum Bayesians who propose no correctness standards whatsoever between physical situations and quantum states. For these QBists, a quantum state just is a person's point of view - it's neither correct nor incorrect, appropriate or inappropriate. Between these two extremes, we have views like Rovelli's, where each physical situation can be described equally well at least two quantum states, depending on one's choice of a direction of time. Some people also think that Bohr was a non-objectivist about quantum states (see Zinkernagel, 2016). However, I find that view hard to square with Bohr's repeated pronouncements of the "objectivity of the quantum-mechanical description".

I propose that we stop talking about the ill-defined notion of quantum state realism, and that we start talking instead about these sorts of question - e.g. whether quantum theory comes with objective standards for the ascription of states to physical situations. First of all, what role do physical situations, described in ordinary language, play in this debate? Could we replace "physical situation" with something more neutral and description-free, such as "object" or "system"? The problem with that suggestion is that the bare notion of an object or a system cannot give us any sort of standard for comparison. For example, we might say: "according to Healey, for each object $X$, there is a unique correct quantum state." But how does Healey individuate objects? If he has different standards for individuating objects than Rovelli has, then their apparently diverging views might in fact agree. Thus, the question of appropriate use of quantum states requires a target, or standard of reference, on which all parties antecedently agree. The notion of a "physical situation" is supposed to offer a plausible standard of reference.

I already suggested a shift from the ontological question "do states ex- 
ist?" to the representational question "how do states represent?" Now I'm suggesting that this representational question be given a normative reading: what are the rules govering the use of quantum states? That, I believe, is the real issue at stake, although it is masked by emotionally charged words such as "ontological status".

There is a second question, closely related to the first one. Should we apply unitary dynamics without exception? Some people say yes (e.g. Bohm, Everett, Wallace), and others say no (e.g. GRW, Rovelli, Healey). But even this disagreement is not as clear-cut as it may seem. Even those who believe in the universal validity of unitary dynamics allow themselves to use "effective states". The "true state", they say, follows unitary dynamics. But for calculational purposes, there can be great advantages to using the effective state.

I'm no verificationist, and so I don't propose that we collapse the distinction between real and effective states. Nonetheless, I'm interested here in the rules for using states, i.e. for deciding whether one ought to use the state that results from unitary evolution, or whether one is permitted to use the state that results from application of the projection postulate. Or to put it in explicitly representational language: the question is whether the state that results from unitary evolution is the only one that is "apt" to one's situation, or whether the state resulting from the projection postulate might also be "apt" to one's situation. Interestingly, all parties seem to agree that the state resulting from the projection posulate is "apt" in some sense. Even the most fervent anti-collapsers will tell you that the projected state is correct for all practical purposes. Then they'll remind you that it's not the "real" state. But I would then ask: not the real state of what? We're back again to the question of how to identify the target $X$ of our representation via a quantum state.

Acknowledgments: Thanks to Eddy Chen for guidance about wavefunction realism, and to Tom Ryckman for sending a preprint of (Ryckman, 2017), which got me interested in Cassirer's view.

\section{References}

Albert, D. Z. (1996). Elementary quantum metaphysics. In Bohmian mechanics and quantum theory: an appraisal, pages 277-284. Springer. 
Alfsen, E. M. and Shultz, F. W. (2012). State spaces of operator algebras: basic theory, orientations, and $C^{*}$-products. Springer.

Belot, G. (2012). Quantum states for primitive ontologists. European journal for philosophy of science, 2(1):67-83.

Bohr, N. (1928). Bohr letter to Christian Møller, 14 June.

Bricker, P. (1996). Properties. In The encyclopedia of philosophy, supplement, pages 469-473. Simon and Schuster MacMillan.

Callender, C. (2015). One world, one beable. Synthese, 192(10):3153-3177.

Carnap, R. (1939). Foundations of logic and mathematics. In Neurath, O., Carnap, R., and Morris, C., editors, International Encyclopedia of Unified Science.

Carroll, S. (2017). The big picture: on the origins of life, meaning, and the universe itself. Dutton.

Cassirer, E. (1955). The philosophy of symbolic forms: language, volume 1. Yale University Press.

Chakravartty, A. (2017). Scientific realism. Stanford online encyclopedia of philosophy.

Chen, E. (2017). Our fundamental physical space: An essay on the metaphysics of the wavefunction. Journal of Philosophy, 114:7.

Chen, E. (2018). Realism about the wave function. Philosophy compass.

Esfeld, M. (2014). Quantum Humeanism, or: physicalism without properties. The Philosophical Quarterly, 64(256):453-470.

Esfeld, M. and Deckert, D.-A. (2017). A minimalist ontology of the natural world. Routledge.

Faye, J. (2014). Copenhagen interpretation of quantum mechanics. Stanford Online Encyclopedia of Philosophy.

Forrest, P. (1988). Quantum metaphysics. Blackwell. 
Frigg, R. and Nguyen, J. (2016). Scientific representation. Stanford online encyclopedia of philosophy.

Goldstein, S. and Zanghì, N. (2013). Reality and the role of the wave function in quantum theory. In Albert, D. Z. and Ney, A., editors, The wave function: essays on the metaphysics of quantum mechanics, pages 91-109. Oxford University Press.

Halvorson, H. (2012). What scientific theories could not be. Philosophy of science, $79(2): 183-206$.

Healey, R. (2015). How pragmatism reconciles quantum mechanics with relativity. 3am Interview. http://www.3ammagazine.com/3am/howpragmatism-reconciles-quantum-mechanics-with-relativity-etc/.

Healey, R. (2016). Quantum-Bayesian and pragmatist views of quantum theory. Stanford Online Encyclopedia of Philosophy.

Healey, R. (2017). The quantum revolution in philosophy. Oxford University Press.

Hirsch, R. J. (2017). From representation to reality: essays on the nature of scientific exegesis. PhD thesis, Princeton University.

Howard, D. (2004). Who invented the "Copenhagen Interpretation"? a study in mythology. Philosophy of Science, 71(5):669-682.

Kadison, R. V. and Ringrose, J. R. (1991). Fundamentals of the theory of operator algebras. American Mathematical Society.

Miller, E. (2014). Quantum entanglement, Bohmian mechanics, and Humean supervenience. Australasian Journal of Philosophy, 92(3):567-583.

Ney, A. (2012). The status of our ordinary three dimensions in a quantum universe. Nô̂s, 46(3):525-560.

Ney, A. and Albert, D. (2013). The wave function: essays on the metaphysics of quantum mechanics. Oxford University Press.

North, J. (2013). The structure of a quantum world. In Albert, D. Z. and Ney, A., editors, The wave function: essays on the metaphysics of quantum mechanics, pages 184-202. Oxford University Press Oxford. 
Penrose, R. (2016). Fashion, faith, and fantasy in the new physics of the universe. Princeton University Press.

Pringe, H. (2014). Cassirer and Bohr on intuitive and symbolic knowledge in quantum physics. Theoria. Revista de Teoría, Historia y Fundamentos de la Ciencia, 29(3):417-429.

Reichenbach, H. (1965). The theory of relativity and a priori knowledge. Univ of California Press.

Rovelli, C. (2016). An argument against the realistic interpretation of the wave function. Foundations of Physics, 46(10):1229-1237.

Ruetsche, L. (2011). Interpreting quantum theories. Oxford University Press.

Ryckman, T. (2017). Cassier and Dirac on the symbolic method in quantum mechanics: a confluence of opposites. Journal for the History of Analytic Philosophy, 6(3):194-224.

Saunders, S. (2010). Many worlds? an introduction. In Many worlds? Everett, quantum theory, and reality, pages 1-. Oxford University Press.

Swanson, N. (2018). How to be a relativistic spacetime state realist. British journal for the philosophy of science.

Van Fraassen, B. C. (1976). To save the phenomena. The Journal of Philosophy, 73(18):623-632.

Wallace, D. (2008). The quantum measurement problem: state of play. In Rickles, D., editor, The Ashgate companion to the new philosophy of physics. Ashgate.

Wallace, D. (2012). The emergent multiverse. Oxford University Press.

Wallace, D. (2013). The Everett interpretation. In The Oxford handbook of the philosophy of physics. Oxford University Press.

Wallace, D. (2018a). Against wavefunction realism. In Weslake, B. and Dasgupta, S., editors, Current controversies in the philosophy of science. Routledge. 
Wallace, D. (2018b). Quantum theory as a framework, and its implications for the quantum measurement problem. In French, S. and Saatsi, J., editors, Realism and the Quantum. Oxford University Press.

Wallace, D. and Timpson, C. G. (2010). Quantum mechanics on spacetime I: Spacetime state realism. The British journal for the philosophy of science, 61(4):697-727.

Zinkernagel, H. (2016). Niels Bohr on the wave function and the classical/quantum divide. Studies in History and Philosophy of Modern Physics, 53:9-19. 